\title{
LOS PROCESOS DE DISEÑO DE TRABAJOS FINALES DE GRADUACIÓN EN LAS UNIDADES ACADÉMICAS DE LA FACULTAD DE CIENCIAS SOCIALES: NORMAS, AVANCES Y DESAFÍOS
}

\author{
DESIGN PROCESSES OF GRADUATION FINAL PAPERS IN THE \\ ACADEMIC UNITS OF THE FACULTY OF SOCIAL SCIENCES: \\ REGULATIONS, PROGRESS AND CHALLENGES
}

\author{
Lisbeth Araya Jiménez ${ }^{1}$ \\ arayalis@gmail.com \\ Nidia Morera Guillén ${ }^{2}$ \\ nidia.morera@ucr.ac.cr
}

\begin{abstract}
Resumen
Este artículo es uno de los primeros resultados materiales y concretos de los recientes esfuerzos de articulación asumidos por las unidades académicas de la Facultad de Ciencias Sociales bajo la convocatoria del Decanato. El artículo da cuenta en primer lugar de la normativa que regula los procesos de diseño, ejecución y presentación de los Trabajos Finales de Graduación (TFG); en segundo lugar se caracteriza de manera concisa la formación académica en investigación en general y los cursos destinados directamente a apoyar los procesos de elaboración de los TFG; el tercer apartado hace un breve análisis comparativo, para concluir con la identificación de los principales fortalezas, debilidades y retos. El artículo finaliza con algunas alternativas de solución a los desafíos expuestos.

Palabras clave: Comisión de Trabajos Finales de Graduación, normas, avances, desafíos, Facultad de Ciencias Sociales.
\end{abstract}

\section{Abstract}

This article is one of the first material and concrete results of recent joint efforts made by the academic units of the Faculty of Social Sciences under the call of the Dean. The article in its first chapter reports current regulations governing the design, execution and presentation of Graduation Final Papers $(G F P)$, at the second chapter concisely characterized academic training in research in general and the courses designed directly to support the process of preparing the GFP, the third section is a brief comparative analysis, concluding with the identification of the main strengths, weaknesses and challenges. The article ends with some alternative solutions to the challenges identified.

Keywords: Graduation Final Papers Commission, regulations, progress, challenges, Faculty of Social Sciences.

\footnotetext{
1 Docente e Investigadora, Coordinadora de la Comisión de Trabajos Finales de Graduación, Escuela de Ciencias de la Comunicación Colectiva, Universidad de Costa Rica, Sede Rodrigo Facio

2 Docente, Directora de la Escuela de Trabajo Social, Directora del Posgrado en Trabajo Social, Universidad de Costa Rica, Sede Rodrigo Facio
} 


\section{Introducción}

El propósito de este artículo es presentar un análisis comparativo e integrador de la normativa, la organización, los retos y desafíos de las Comisiones de Trabajos Finales de Graduación de las Unidades Académicas de la Facultad de Ciencias Sociales en el cumplimiento de su responsabilidad en la organización y desarrollo de los procesos de diseño, aprobación, ejecución y presentación de los trabajos finales de graduación.

Se elaboró con base en la información oral y escrita aportada por las y los participantes en el primer y segundo taller de TFG realizado en la Facultad de Ciencias Sociales los días 9 y 23 de abril del 2013, cuyo propósito inicial fue compartir: a) los mecanismos mediante los cuales cada unidad académica desarrolla la importante tarea de acompañar al estudiantado en el diseño, la aprobación, la ejecución y la presentación de sus trabajos finales de graduación, con énfasis en determinar los avances, retos y desafíos que se enfrentan: b) aprendizajes y experiencias y c) discutir alternativas de solución conjunta.

Es importante señalar que en los talleres de TFG indicados participaron representantes de las escuelas de Antropología, Ciencias de la Comunicación Colectiva, Ciencias Políticas, Geografía, Historia-Archivística, Psicología, Sociología y Trabajo Social.

Previo a la realización del taller se envió a cada unidad académica una guía de trabajo que tomó en cuenta: a) los cursos preparatorios para la elaboración de los TFG con énfasis en su contenido, objetivos, productos, vínculo con la Comisión de TFG, fortalezas y debilidades y, b) la caracterización de las Comisiones de Trabajos Finales de Graduación con acento en su composición, normativa, instrumentos, procedimientos para evaluar las propuestas, vínculos con los comités asesores; experiencias de diseños de TFG interdisciplinarios, principales problemáticas y propuestas de mejora.

La estructura del artículo da cuenta en primer lugar de la normativa que regula los procesos de diseño, ejecución y presentación de los TFG; en segundo lugar se caracteriza brevemente la formación académica en investigación en general y los cursos destinados directamente a apoyar los procesos de elaboración de los TFG; el tercer apartado hace un breve análisis comparativo, para concluir con la identificación de los principales fortalezas, debilidades y retos, el artículo finaliza con algunas alternativas de solución a los desafíos expuestos.

\section{Normativa que regula los procesos de diseño y aprobación de propuestas de TFG}

La Vicerrectoría de Docencia, en la circular VD-C-16-2012, relacionada con las comisiones institucionales, hace referencia a los aspectos a considerar en la conformación y el funcionamiento de las comisiones institucionales. Particularmente señala que las comisiones ordinarias permanentes más comunes son:
a) Evaluación, Orientación, Selección y Matrícula
b) Currículo, Credenciales y Reconocimiento
c) Trabajos Finales de Graduación
d) Investigación
e) Acción Social

La normativa a la cual hace referencia esta circular establece que las Comisiones estarán conformadas por un mínimo de tres docentes y un máximo de cinco, uno de los cuales debe fungir como coordinador o coordinadora. También señala que la carga académica que se debe asignar a cada docente será de 0 a 5 horas reloj y para la persona que coordine de 0 a 10 horas reloj.

Algunas comisiones ordinarias están regidas por reglamentos específicos tales como el Reglamento de Trabajos Finales de Graduación en el caso de la Comisión de Trabajos Finales de Graduación y el Reglamento de Régimen Académico Estudiantil en el caso de la Comisión de Evaluación, Orientación, Selección y Matrícula.

Las comisiones permanentes coadyuvan con las tareas de la Dirección y tienen funciones permanentes y similares en la mayoría de las Unidades Académicas de la Facultad de Ciencias Sociales y de la Universidad de Costa Rica.

Dadas sus características y funciones, algunas de ellas requieren un trabajo continuo y voluminoso, que justifica la asignación de carga académica para sus integrantes. Sin embargo, en los casos en que a estas Comisiones no se les fije 
carga académica, corresponde a la persona que dirige cada Unidad Académica decidir sobre la asignación de dichas cargas.

Como se dijo, en particular las Comisiones de Trabajos Finales de Graduación se rigen por el Reglamento de Trabajos Finales de Graduación, específicamente por lo indicado en los Artículos del 25 al 30, que se parafrasean a continuación.

Al respecto en el Artículo 25 se establece que "Cada Unidad Académica tendrá una Comisión de Trabajos Finales de Graduación nombrada por la persona que ocupe el cargo de Directora, la cual estará integrada por personal docente debidamente inscrito en Régimen Académico y con el título mínimo de licenciatura. Esta comisión se nombrará por un período de dos años renovables y sus decisiones son apelables ante la Dirección de la unidad académica respectiva, como instancia inmediata y única"

En el Artículo 26 se plantea que "Toda solicitud para llevar a cabo un trabajo final de graduación, según las opciones contempladas en cada plan de estudio, deberá ser resuelta por la Comisión de Trabajos Finales de Graduación de la respectiva unidad académica. Una vez aprobado el plan, la responsabilidad de llevarlo a cabo de modo satisfactorio será del candidato o la candidata, bajo la guía y supervisión del Comité Asesor designado para tal efecto".

El Artículo 27 establece que "En cada Unidad Académica la Comisión de Trabajos Finales de Graduación debe velar por el cumplimiento de las normas establecidas en el reglamento, igualmente, deberá evaluar periódicamente el sistema de graduación."

El Artículo 28 se señala que "Las decisiones que debe adoptar la Comisión de Trabajos Finales de Graduación se tomarán por simple mayoría".

Los artículos 29 y 30 plantean que las y los integrantes del Comité Asesor que se designen por parte de la Dirección de cada unidad académica para cada Trabajo Final de Graduación, deben ser por lo menos licenciados y estar en Régimen Académico. La persona que se nombre como directora deberá poseer la categoría de Adjunta o Superior. En casos justificados, la persona que ocupa el puesto de Dirección de la Escuela, en consulta con la Comisión de Trabajos Finales de Graduación, podrá levantar los requisitos de estar en Régimen Académico y de poseer la categoría de Adjunto o Superior, así como también integrar al Comité Asesor personas calificadas que no tengan una relación formal con la Universidad, pero que tengan los méritos académicos necesarios para dirigir un Trabajo Final de Graduación o para formar parte del Comité.

Interesa destacar que cada unidad académica dispone de normas y regulaciones particulares para orientar al estudiantado y al personal docente en lo que se refiere a los procesos de elaboración, evaluación, ejecución y presentación de los Trabajos Finales de Graduación, algunas de las cuales se detallan a continuación:

- La Escuela de Antropología cuenta con una página WEB en la cual indica la conformación y las tareas de la Comisión de TFG. Además se informa acerca de los requisitos para presentar proyectos de TFG.

- La Carrera de Archivística cuenta con un documento con indicadores para la evaluación de los proyectos de Trabajos Finales de Graduación y además tiene un documento que da cuenta de los lineamientos para realizar TFG modalidad práctica dirigida.

- La Escuela de Ciencias de la Comunicación Colectiva dispone en la página WEB de una guía para la elaboración (apartados a considerar y requisitos de sus contenidos), presentación y trámite de los anteproyectos ante la comisión de TFG, así como de una nota donde se establecen los criterios a tomar en cuenta para que un o una docente dictamine una propuesta de TFG.

- La Escuela de Psicología en su sitio WEB cuenta con un instructivo para la presentación, evaluación y aprobación por parte de la Comisión de Trabajo Finales de Graduación, de las propuestas. Este documento incluye consideraciones generales de la Comisión de TFG, requisitos para presentar propuestas, procedimientos de evaluación, las modalidades y como son entendidas en la Unidad Académica. También incluye como anexos los aspectos de forma por modalidad.

- La Escuela de Sociología tiene una guía básica para elaborar y presentar los diseños en la cual se exponen con detalle cada uno de los componentes a incluir por opción de TFG, así como aspectos de forma tales como 
extensión y tipo de letra. En su sitio WEB tiene información respecto a los requisitos para entregar diseños, una guía básica de diseño, las opciones de TFG y la fórmula para recepción de propuestas.

La Escuela de Trabajo Social dispone de guías específicas para formular propuestas de TFG según modalidad y rúbricas de evaluación para cada una. También en la página WEB se informa acerca de las orientaciones para presentar propuestas, las cuales incluyen aspectos de extensión, formato y número de ejemplares, asî como los criterios generales para su evaluación.

\section{Breve caracterización de la formación académica en investigación en general y para la formulación de propuestas de Trabajos Finales de Graduación por unidades académicas}

En los cuadros que se presentan seguidamente se consignan según Unidad Académica, los cursos vinculados con la formación en materia de investigación, así como los cursos que se relacionan directamente con apoyar la elaboración y ejecución de los trabajos finales de graduación. Al respecto es importante señalar que todas las Escuelas cuentan con formación de base en materia de investigación.

La información consignada en este apartado se obtuvo de las matrices aportadas por las y los directores de cada una de las Escuelas que conforman la Facultad de Ciencias Sociales, en los talleres realizados para discutir posibilidades de articulación interdisciplinaria.

Estos mismos cuadros se utilizaron en el artículo titulado La formación en investigación social en la Facultad de Ciencias Sociales de la Universidad de Costa Rica: diversidad disciplinaria y posibilidades de articulación en función de la excelencia académica, en ese caso el análisis enfatizó en la formación en investigación, para los efectos del presente artículo los Trabajos Finales de Graduación ocupan la centralidad de las reflexiones.

Cuadro 1

Cursos relacionados con la formación en investigación y con elaboración de trabajos finales de graduación, Antropología

\begin{tabular}{|c|c|c|c|c|}
\hline $\begin{array}{l}\text { Unidad } \\
\text { Académica }\end{array}$ & Cursos & Créditos & Nivel & Naturaleza \\
\hline \multirow[t]{17}{*}{ Antropología } & Métodos y técnicas de investigación antropológica & 4 & II & Introductorio \\
\hline & $\begin{array}{l}\text { Métodos y técnicas de investigación en Antropología } \\
\text { Social }\end{array}$ & 6 & III & \\
\hline & Métodos y técnicas de investigación en Arqueología & 6 & III & \\
\hline & Problemas contemporáneos de la antropología social & 3 & $\mathrm{~V}$ & Teórico \\
\hline & $\begin{array}{l}\text { Seminario crítico de la investigación Arqueológica en } \\
\text { Costa Rica. }\end{array}$ & 3 & $\mathrm{~V}$ & \\
\hline & Técnicas avanzadas en la gestión y evaluación social & 4 & $\mathrm{~V}$ & Teórico-práctico \\
\hline & Análisis e interpretación de datos en antropología social & 3 & $\mathrm{~V}$ & \\
\hline & Investigación Arqueológica I & 6 & $\mathrm{~V}$ & \\
\hline & Investigación Arqueológica II & 6 & $\mathrm{~V}$ & \\
\hline & Técnicas avanzadas en arqueología & 3 & $\mathrm{~V}$ & \\
\hline & Taller de diseño de investigación & 3 & IV & Trabajo Final de Graduación \\
\hline & Taller de Antropología Social I & 8 & $\mathrm{~V}$ & \\
\hline & Taller de antropología Social II & 8 & $\mathrm{~V}$ & \\
\hline & Técnicas Avanzadas en la Investigación Socio-Cultural & 4 & $\mathrm{~V}$ & \\
\hline & Taller de Arqueología I & 6 & $\mathrm{~V}$ & \\
\hline & Taller de Arqueología II & 6 & $\mathrm{~V}$ & \\
\hline & Total de créditos & 75 & & \\
\hline
\end{tabular}

Fuente: Elaboración propia. 
Cabe señalar que en Antropología, para el dos opciones de especialización: Antropología nivel de licenciatura las y los estudiantes poseen Social o Arqueología.

Cuadro 2

Cursos relacionados con la formación en investigación y con elaboración de trabajos finales de graduación, Ciencias de la Comunicación Colectiva

\begin{tabular}{lllll}
\hline Unidad Académica & Cursos & Créditos & Nivel & Orientación \\
\hline Ciencias de la & Análisis del Discurso & 3 & I & \\
\cline { 2 - 5 } $\begin{array}{l}\text { Comunicación } \\
\text { Colectiva }\end{array}$ & Construcción del objeto de investigación & 3 & II & Introductorio \\
\cline { 2 - 5 } & Investigación de públicos y audiencias & 3 & & Teórico \\
\cline { 2 - 5 } & Diseños, construcción de datos y análisis cuantitativos & 3 & II & Teórico-práctico \\
\cline { 2 - 5 } & Diseños, construcción de datos y análisis cualitativos & 3 & II & \\
\cline { 2 - 5 } & Taller de Investigación 1 & 3 & V & Trabajo Final de Graduación \\
\cline { 2 - 5 } & Taller de Investigación 2 & 3 & V & Trabajo Final de Graduación \\
\cline { 2 - 5 } & Total de créditos & 21 & & \\
\hline
\end{tabular}

Fuente: Elaboración propia.

La Escuela de Ciencias de la Comunicación Colectiva ofrece cuatro modalidades de especialización: Periodismo, Producción Audiovisual,
Publicidad y Relaciones Públicas, en consecuencia, las y los estudiantes, pueden realizar sus TFG en cualquier de ellas.

Cuadro 3

Cursos relacionados con la formación en investigación y con elaboración de trabajos finales de graduación, Ciencias Políticas

\begin{tabular}{llccl}
\hline Unidad Académica & Cursos & Créditos & Nivel & Orientación \\
\hline Ciencias Políticas & Análisis Político Cuantitativo & - & II & Teórico-práctico \\
\cline { 2 - 5 } & Técnicas Cualitativas de Investigación & - & II & \\
\cline { 2 - 5 } & Metodología & - & II & \\
\cline { 2 - 5 } & Análisis e Interpretación de Datos Políticos & - & III & \\
\cline { 2 - 5 } & Teoría y Lógica de la Ciencia Política & V & Teórico \\
\cline { 2 - 5 } & Epistemología de la Ciencia Política & V & Teórico \\
\cline { 2 - 5 } & Seminario de Metodología & V & Trabajo Final de Graduación \\
\cline { 2 - 5 } & Taller de Investigación & V & Trabajo Final de Graduación \\
\cline { 2 - 5 } & Total de créditos & - & & \\
\hline
\end{tabular}

Fuente: Elaboración propia.

Cuadro 4

Cursos relacionados con la formación en investigación y con elaboración de trabajos finales de graduación, Geografía

\begin{tabular}{llccl}
\hline Unidad Académica & Cursos & Créditos & Nivel & Orientación \\
\hline \multirow{2}{*}{ Geografía } & Investigación Geográfica & 3 & II & Teórico-práctico \\
\cline { 2 - 5 } & Teoría y Métodos & 4 & V & Trabajo Final de Graduación \\
\cline { 2 - 5 } & Elaboración y evaluación de proyectos & 4 & V & Trabajo Final de Graduación \\
\cline { 2 - 5 } & Total de créditos & 11 & & \\
\hline
\end{tabular}

Fuente: Elaboración propia. 
Cuadro 5

Cursos relacionados con la formación en investigación y con elaboración de trabajos finales de graduación, Historia

\begin{tabular}{|c|c|c|c|c|}
\hline Unidad Académica & Cursos & Créditos & Nivel & Orientación \\
\hline \multirow[t]{11}{*}{ Historia } & Introducción a la Historia y técnicas para su estudio I & 3 & $\mathrm{I}$ & Introductorio \\
\hline & Introducción a la Historia y técnicas para su estudio II & 3 & I & \\
\hline & Seminario temático & 5 & IV & \\
\hline & Métodos de investigación histórica I & 3 & III & \\
\hline & Métodos de investigación histórica II & 3 & III & \\
\hline & Taller de divulgación histórica & 4 & IV & \\
\hline & Taller de investigación histórica I & 3 & IV & Trabajo Final de Graduación \\
\hline & Taller de investigación histórica II & 3 & $\mathrm{~V}$ & \\
\hline & Taller de investigación I & 5 & $\mathrm{~V}$ & \\
\hline & Taller de investigación II & 5 & $\mathrm{~V}$ & \\
\hline & Total de créditos & 54 & & \\
\hline \multirow{5}{*}{$\begin{array}{l}\text { Carrera } \\
\text { Archivística }\end{array}$} & HA 2083 Métodos de Investigación I & 3 & & \\
\hline & HA2084 Métodos de Investigación II & 3 & & \\
\hline & Temas y Problemas para Investigación 3 & 3 & & Trabajo Final de Graduación \\
\hline & Taller de Graduación & 3 & & Trabajo Final de Graduación \\
\hline & Total de créditos & & & \\
\hline
\end{tabular}

Fuente: Elaboración propia.

Cuadro 6

Cursos relacionados con la formación en investigación y con elaboración de trabajos finales de graduación, Psicología

\begin{tabular}{llccl}
\hline Unidad Académica & Cursos & Créditos & Nivel & Orientación \\
\hline \multirow{2}{*}{ Psicología } & Investigación I: Teoría del método & 2 & I & Introductorio \\
\cline { 2 - 5 } & Investigación II: Construcción del dato & 3 & II & Teórico-práctico \\
\cline { 2 - 5 } & Investigación III: Métodos y diseños de investigación & 2 & II & \\
\cline { 2 - 5 } & Investigación IV: Tratamiento de datos cualitativos & 3 & III \\
\cline { 2 - 5 } & Investigación V: Análisis descriptivo e inferencial & 2 & III \\
\cline { 2 - 5 } & Investigación VI: Análisis multivariado & 2 & IV \\
\cline { 2 - 5 } & Investigación VII: Construcción de pruebas y psicometría & 3 & IV \\
\cline { 2 - 5 } & Investigación VIII: Anteproyecto de TFG & 3 & V & Trabajo Final de Graduación \\
\cline { 2 - 4 } & Total de créditos & 20 créditos & \\
\hline
\end{tabular}

Fuente: Elaboración propia. 
Cuadro 7

Cursos relacionados con la formación en investigación y con elaboración de trabajos finales de graduación, Sociología

\begin{tabular}{|c|c|c|c|c|}
\hline Unidad Académica & Cursos & Créditos & Nivel & Orientación \\
\hline \multirow[t]{10}{*}{ Sociología } & Metodología e investigación sociológica I & 3 & $\mathrm{I}$ & Introductorio \\
\hline & Metodología e investigación sociológica II & 3 & $\mathrm{I}$ & \\
\hline & Técnicas Cualitativas de Investigación Social & 3 & II & Teórico-práctico \\
\hline & Técnicas de Manejo de la Información & 3 & II & \\
\hline & Técnicas Cuantitativas de Investigación Social & 3 & III & \\
\hline & Gestión de la Investigación y la Acción Social I & 3 & IV & \\
\hline & Gestión de la Investigación y la Acción Social II & & IV & \\
\hline & Taller Integrado de Investigación Sociológica I & 3 & IV & Trabajo Final de Graduación \\
\hline & Taller Integrado de Investigación Sociológica II & 3 & IV & \\
\hline & Total de créditos & 24 & & \\
\hline
\end{tabular}

Fuente: Elaboración propia.

Cuadro 8

Cursos relacionados con la formación en investigación y con elaboración de trabajos finales de graduación, Trabajo Social

\begin{tabular}{llrll}
\hline Unidad Académica & Cursos & Créditos & Nivel & Orientación \\
\hline Trabajo Social & Teoría y Métodos en Investigación Social I & 3 & III & Introductorios, teóricos, metodológicos \\
\cline { 2 - 5 } & Teoría y Métodos en Investigación Social II & 3 & III & \\
\cline { 2 - 5 } & Epistemología I & 3 & II & Teórico-metodológicos \\
\cline { 2 - 5 } & Epistemología II & 3 & II & \\
\cline { 2 - 5 } & Epistemología III & 3 & IV & \\
\cline { 2 - 5 } & Investigación evaluativa I & 2 & V & \\
\cline { 2 - 5 } & Investigación evaluativa II & 3 & V & \\
\cline { 2 - 5 } & Diseño de trabajo final de graduación & 4 & V & Trabajo Final de Graduación \\
\cline { 2 - 5 } & Total de créditos & 24 & & \\
\hline
\end{tabular}

Fuente: Elaboración propia. 
En resumen y orden decreciente, la información consignada en los cuadros anteriores da cuenta de lo siguiente:

a) Escuela de Antropología: es la que cuenta con mayor cantidad de cursos en materia de investigación con un total de 16 , de los cuales tres son introductorios o básicos en materia de investigación para la formación del estudiantado con énfasis en la disciplina antropológica; ocho tienen relación con el diseño y el avance en la ejecución del trabajo final de graduación para el énfasis en Antropología Social y siete se relacionan con el diseño del TFG del énfasis de Arqueología.

b) Escuela de Historia: tiene catorce cursos de los cuales en ocho se abordan temas tales como la introducción a la Historia como disciplina y las técnicas para su estudio, qué es la Historia y qué es la Historiografía, fundamentos epistemológicos de la investigación en Historia, especificidades del conocimiento desde la Historia, aportes de distintos paradigmas, el método y las fuentes en la Historia, métodos y técnicas de investigación cualitativas y cuantitativas. Un total de cuatro cursos aportan elementos para la formulación del diseño del TFG y para la elaboración de dos capítulos al concluir el $\mathrm{V}$ año.

c) Escuela de Sociología: tiene un total de nueve cursos en materia de investigación que abordan la metodología de investigación sociológica, en la cual se discuten aspectos conceptuales básicos de investigación social, la caracterización del pensamiento científico social con respecto a los orígenes, alcances del conocimiento y las diferentes perspectivas metodológicas en que se sustenta. También hay cursos de técnicas de investigaciones cualitativas y cuantitativas, técnicas de manejo de la información y cursos de gestión de la investigación social. Se cuenta con dos cursos tipo taller en los cuales se formulan los diseños de TFG.

Las Escuelas de Ciencias Políticas, Psicología y Trabajo Social cuentan con ocho cursos de investigación, a saber: d) La Escuela de Ciencias Políticas tiene ocho cursos de investigación orientados al análisis político cuantitativo, a las técnicas cualitativas de investigación, la metodología en las Ciencias Sociales, la metodología para la investigación política, el análisis y la interpretación de datos políticos, teoría, lógica y epistemología de la Ciencia Política. En quinto año se ofrecen dos cursos un seminario de metodología y un Taller de investigación para el diseño y avance en la ejecución del TFG.

e) La Escuela de Trabajo Social cuenta con ocho cursos de investigación. De ellos tres son de epistemología, en los que se contextualiza desde una perspectiva socio histórica de la naturaleza del conocimiento, la filosofía y su relación con las Ciencias Sociales y con el Trabajo Social. En ellos se estudian los paradigmas ontológicos y epistemológicos, con las respectivas concepciones de realidad y de su aprehensión, la relación sujeto-objeto. Se discute el abordaje ontológico vs el abordaje epistemológico de los objetos de estudio y sus implicaciones. También se cuenta con dos cursos de teoría y métodos de investigación en los cuales se estudia la investigación en las ciencias sociales, desde las corrientes positivista, interpretativas y del materialismo dialéctico. Se discuten los métodos de investigación cualitativos y cuantitativos, así como la investigación en Trabajo Social con énfasis en sus objetos, fines y relevancia. También en ellos se estudia cómo elaborar un estado del arte, la formulación del objeto y su problematización, la definición de objetivos, el fundamento teórico-metodológico con los respectivos recursos técnicos instrumentales. En el nivel de licenciatura se dispone de un curso en el cual se acompaña al estudiantado en el proceso de elaboración del diseño de su TFG. Finalmente se cuenta con dos cursos de investigación evaluativa en los cuales se elaboran diseños de investigación evaluativa para programas, proyectos o servicios sociales.

f) Escuela de Psicología: tiene un total de ocho cursos de los cuales siete abordan aspectos tales como la teoría del método, la construcción del dato, los diseños de investigación, 
procedimientos analíticos de la información cualitativa, análisis descriptivo inferencial, análisis multivariado y construcción de pruebas psicométricas, lo cual muestra un énfasis en la investigación cuantitativa centrada en la construcción, obtención, registro y análisis del dato. Por otra parte, se cuenta con un curso para apoyar directamente la elaboración del anteproyecto de TFG.

g) Escuela de Ciencias de la Comunicación Colectiva: cuenta con siete cursos en su plan de estudios relacionados con la formación en investigación, en los cuales se aborda la construcción del objeto de investigación, las perspectivas de la investigación, tipos de estudios en comunicación, lo ético y lo político en la investigación en comunicación, las perspectivas positivistas y constructivistas en investigación social y comunicación; modelos y diseños de construcción de datos y análisis cuantitativos y cualitativos, el proceso de investigación con todos sus componentes, tendencias teóricas de la investigación en comunicación, técnicas cualitativas y cuantitativas de investigación tales como observación, entrevistas, etnografía virtual, técnicas proyectivas, procesamiento y análisis de información, análisis de discursos, investigación de públicos y audiencias. En cuanto al Diseño de TFG cuenta con dos Talleres de Investigación en el quinto año de la carrera.

h) La Escuela de Geografía tiene tres cursos de investigación: uno referido a la investigación geográfica en el cual se abordan las áreas y temas de investigación geográfica, el por qué investigar, qué es investigar, otro relacionado con las etapas de un diseño, la ética en la investigación y los aspectos formales para presentar un proyecto de investigación. Para el TFG se cuenta con un curso de teoría y métodos en quinto año.

En los siguientes apartados, se consignan las fortalezas y debilidades identificadas por las Comisiones para los procesos de diseño, aprobación, ejecución y presentación de los TFG; así como los retos más sentidos. El artículo cierra con propuestas, alternativas de solución concretas susceptibles de implementación.

\section{Fortalezas y debilidades}

\subsection{Fortalezas}

\subsubsection{Procedimientos para recibir y evaluar anteproyectos}

Algunas Comisiones (sociología, psicología por ejemplo) han construido una fórmula para la recepción de diseños que regula tanto los aspectos de contenido como de forma, esto con el objetivo de tener una especie de filtro en las secretarías de las unidades académicas, de modo que los diseños que cumplan con los requisitos pasen a las Comisiones y los que no, sean devueltos a las y los estudiantes para ser debidamente completados.

Los procedimientos seguidos por las Comisiones de TFG de las Escuelas de Antropología, Ciencias de la Comunicación, Ciencias Políticas, Geografía, Historia-Archivística, Psicología, Sociología y Trabajo Social para la revisión y evaluación de los anteproyectos se pueden resumir en:

- La Comisión en pleno revisa el anteproyecto (Sociología),

- Un/a integrante de la Comisión lo revisa en atención a sus ámbitos de trabajo, experiencia o especialización (Psicología)

- La Comisión lo remite a un/a docente que funciona como dictaminador/a externo (Comunicación).

En todos los casos, la revisión y decisión final sobre la aprobación o no del diseño es resorte de la Comisión, de igual forma el tiempo que demora el proyecto desde que se recibe en la unidad académica hasta que se comunica la resolución de la Comisión es de un mes. En todos los casos la Comisión convoca y comunica directamente a la o el estudiante la resolución.

Algunas de las comisiones han implementado como medida de confidencialidad y objetividad (Ciencias de la Comunicación Colectiva y Ciencias Políticas) omitir de las comunicaciones entre estudiantes y persona dictaminadora las identidades de ambos, así, ni las/los sustentantes 
conocen la identidad de quien evalúa su anteproyecto, ni la persona dictaminadora sabe quiénes son las y los postulantes.

Existen con pequeñas variantes dos tipos de dictámenes: 1. Aprobado (algunas comisiones han generado tipologías como "con correcciones menores", "con correcciones mayores"); en esta categoría se incluyen los anteproyectos que se aprueban tal cual y aquellos que se aprueban con modificaciones) 2. Improbado.

\subsubsection{Creación de protocolos y procedimientos}

Una vez revisado en detalle el material aportado por las Unidades Académicas de la Facultad de Ciencias sociales es claro que las Comisiones, a falta de normativa actualizada y precisa (sobre este tema se volverá más adelante en el apartado de alternativas de solución), han tomado la iniciativa y han generado fórmulas, guías, lineamientos e instructivos en procura de cumplir con los objetivos y tareas de su competencia y de acompañar, de la mejor manera posible al estudiantado en sus procesos de investigación (diseño, ejecución, análisis, elaboración de los documentos finales y presentación). Esta es sin duda una fortaleza. La debilidad estriba en el hecho de que cada Escuela ha recorrido el camino en solitario, encontrado en él, básicamente, las mismas piedras que su vecino/a; es interesante encontrar además, que las grúas, las palas y los picos, las catapultas (proporcionales al tamaño de la piedra) están hechas también, estructuralmente, del mismo material.

En general se pudo determinar que todas las Escuelas se han venido preocupando por clarificar en el marco del Reglamento de TFG: a) la definición de cada modalidad de TFG, b) los aspectos a considerar en las propuestas según modalidad, c) los requisitos de contenido y de forma para presentar ante la Comisión una propuesta y, d) los criterios y procedimientos para evaluar las propuestas según modalidad.

\subsubsection{Vinculación entre cursos de investigación y Comisiones de Trabajos Finales de Graduación}

Algunas de las Escuelas (como Ciencias Políticas y Ciencias de la Comunicación
Colectiva) han integrado sus Comisiones con las y los docentes de los cursos preparatorios al TFG, esto permite tener un conocimiento cercano de los temas, líneas de investigación y estrategias metodológicas más usadas, así como identificar las falencias y requerimientos particulares en el proceso de investigación, de modo que se pueden prever y atender oportunamente necesidades del estudiantado. Esta relación directa entre la Comisión y los cursos de investigación existentes en los planes de estudio para elaborar el TFG, ha permitido abordar consultas y generar soluciones en correspondencia con la normativa y procedimientos establecidos por ejemplo, en materia de ética y consentimiento informado.

\subsubsection{Estrategias para acortar tiempos de graduación}

Aunque se identifican similitudes entre las unidades académicas, también se encontraron avances cualitativos importantes en algunas de ellas que pueden ser de mucha utilidad para otras unidades académicas en torno al importante proceso de cierre de la formación académicaprofesional del estudiantado de la Facultad de Ciencias Sociales.

Algunas de las estrategias que se lograron identificar en este sentido se detallan a continuación:

a) Procurar la selección del comité asesor desde que el o la estudiante inicia el diseño del TFG:

En las Escuelas de Ciencias de la Comunicación Colectiva, Trabajo Social y Ciencias Políticas, por ejemplo, durante el primero de los cursos para elaborar el diseño del TFG, se invita a las y los estudiantes a identificar posibles docentes que podrían conformar su Comité Asesor. Esto con el objetivo de que el Comité Asesor (que será formalmente nombrado hasta que el diseño sea aprobado por la Comisión de TFG) se involucre con el/la estudiante, desde la selección primera del tema, la construcción de objetivos y la propuesta de abordaje (teórico y metodológico). 
b) Enlazar los cursos en los cuales se acompaña en la elaboración de los diseños de TFG con el trabajo de la Comisión:

Por ejemplo, en Ciencias Políticas si el o la estudiante aprueba el curso preparatorio con un anteproyecto que obtiene una nota de 9 o superior y ha expuesto su proyecto ante un tribunal (como ejercicio pedagógico), se le aprueba el anteproyecto en la Comisión de TFG.

c) Algunas unidades académicas tales como Antropología, Ciencias Políticas, Sociología e Historia, cuentan con cursos en los cuales se inicia el diseño de TFG desde el cuarto año del plan de estudio. Ello permite que el estudiantado avance significativamente en la construcción de sus propuestas, e incluso que elaboren algunos capítulos de sus investigaciones, las cuales se aprueban antes de concluir el plan de estudios. Indudablemente esta estrategia permite acortar tiempos de graduación con calidad y excelencia académica.

\subsection{Debilidades}

\subsubsection{Debilidades metodológicas previas}

Una de las debilidades identificadas por varias de las Escuelas de la Facultad son las dificultades que enfrentan las y los estudiantes en la elaboración de su TFG por deficiencias metodológicas previas. Pese a los ingentes esfuerzos y la multiplicidad de cursos de investigación presentes en los planes de estudio de las Escuelas de la Facultad, como se discutió unas líneas más arriba, al parecer, las deficiencias metodológicas no han sido superadas del todo.

\subsubsection{Tiempo reducido para la elaboración del diseño}

Algunas de las Comisiones señalan como una debilidad la escasez de tiempo disponible para que las y los estudiantes elaboren el diseño del TFG, pues en la mayoría de las unidades académicas se cuenta con un semestre, máximo dos, para realizar esta tarea.

\subsubsection{Falta de inducción y relación entre las Comisiones de TFG y Comités Asesores}

En general se identifica como una debilidad la falta de inducción y de relación entre la Comisión de TFG y los Comités Asesores. Los vínculos suelen ser únicamente los formales, es decir de asesoría, consulta y trámites; pero se carece de un diálogo cercano y permanente que permita llevar los ritmos de avance y necesidades específicas durante la elaboración de los TFG.

\section{Retos}

\subsection{Designación de cargas docentes}

En algunas unidades académicas, en atención a la escasez de tiempos se opta por ubicar todos los recursos en tiempos docentes, de manera que la Escuela (y/o la Comisión) acaba por solicitar trabajo ad honorem, tanto para algunos/ as miembros de las Comisiones de TFG como para miembros de los Comités Asesores. Esto es un reto en tanto el acompañamiento a cualquier modalidad de TFG debe reconocerse y valorarse como la labor académica que supone.

\subsection{Divulgacióndenormativayprocedimientos}

En no pocos casos, pese a la elaboración de guías, lineamientos e instructivos, estos no son conocidos y atendidos de manera oportuna por la comunidad estudiantil.

Uno de los retos compartidos por algunas de las Unidades Académicas de la Facultad es el cumplimiento de la normativa en lo que a los tiempos de matrícula de Investigación Dirigida, Práctica Dirigida o Seminario de Graduación, corresponde, una vez aprobado el anteproyecto. Algunos/as estudiantes, no matriculan en el semestre inmediatamente posterior a la aprobación del anteproyecto, como reza la normativa, o, simplemente, no dan seguimiento a los procesos de matrícula. El problema se da cuando no se comunican formalmente a la Comisión las razones para no seguir con el debido proceso, o bien acogerse al artículo 27 del Reglamento de Régimen Estudiantil y solicitar la "interrupción de estudios". Una de las consecuencias de dicho 
incumplimiento es el cierre de expedientes (al cabo de tres ciclos lectivos, cuatro en casos extraordinarios) que lleva a las y los estudiantes, en consecuencia, a iniciar un nuevo proyecto.

\subsection{Promoción de las diversas modalidad de TFG}

Aún cuando no se cuenta con una estadística que brinde el dato exacto, pues algunas unidades académicas no cuentan con las bases de datos actualizadas, la experiencia de las y los integrantes de las Comisiones por un lado, y los datos en aquellas escuelas que han construido la información de manera sistemática, por otro, dan cuenta de que la mayoría de TFG en la Facultad son Tesis, seguidas de Seminarios, Proyectos de Graduación (en algunas Escuelas este orden entre segundo y tercer lugar se invierte) y en menor medida Prácticas Dirigidas. Así, el reto identificado supone la promoción de las opciones que las y los estudiantes no están considerando (o están considerando en muy pocos casos) para elaborar su TFG, de modo que el abanico disponible se aproveche. Los integrantes de las Comisiones de TFG de la Facultad, han planteado como hipótesis: a) el hecho de que las y los actuales docentes en su gran mayoría elaboraron tesis como TFG por lo que no conocen desde lo vivencial las otras modalidades, y en consecuencia no promueven su elaboración, b) en el imaginario colectivo, la tesis se concibe como él proyecto de graduación, en tanto las otras modalidades gozan de menor prestigio.

\subsection{Relación entre realidad nacional/ mercado laboral y temas de investigación propuestos por el estudiantado}

Parece ser una constante la necesidad de lograr un vínculo más estrecho y directo de los temas de investigación propuestos por el estudiantado con la realidad nacional y los mercados laborales. Esto significa para las Comisiones y para las Unidades Académicas un reto sentido, la formación académica, especialmente en el nivel de la licenciatura y en el proceso de cierre que implica el TFG tendría que convertirse en un mecanismo (aunque claramente no el único ni el primero) que engarce con los retos que enfrenta la sociedad costarricense, en las más diversas materias, atravesando grupos sociales, zonas geográficas, modelos organizativos, y visiones de mundo. Los TFG de la Facultad de Ciencias Sociales deberían ser un espacio para la reflexión y la lectura de las actuales preocupaciones de los diversos colectivos, pero también de sus deudas estructurales, para pensar sus conflictos y desencuentros, para proponer alternativas de abordaje a los retos sociales, económicos, políticos, que obstaculizan y limitan la existencia de una sociedad más justa y equitativa, más solidaria y responsable social, ambiental y económicamente hablando, menos desigual, de mayor bienestar colectivo y menos intereses particulares, una investigación que con perspectiva histórica y visión de futuro, aporte reflexión, sistematicidad, mirada crítica, pero que especialmente sueñe nuevas rutas para transitar en el contexto actual, en sus contradicciones y posibilidades.

\subsection{Articulación entre investigación y acción social}

Sin duda alguna al igual que para la Universidad como un todo sigue siendo necesario articular la docencia, la investigación y la acción social, en el marco de los TFG es impostergable vincular los dos últimos, esto para lograr por un lado un mayor o mejor resultado (o impacto, según corresponda) de los proyectos que se llevan a cabo, ampliar los ámbitos de cobertura, y por otro lado para innovar en las estrategias de abordaje, construir nuevas metodologías o bien para aportar alternativas cada vez más integrales a los problemas sociales mediante la investigación y/o el desarrollo de proyectos.

\section{Alternativas de solución}

\subsection{Actualización de la normativa Reglamento TFG}

Todas las Comisiones que participaron de los talleres que dan origen a este artículo, a saber Antropología, Ciencias de la Comunicación Colectiva, Ciencias Políticas, Geografía, Historia-Archivística, Psicología, Sociología y 
Trabajo Social concuerdan en que una primera prioridad en las soluciones requeridas es la actualización del Reglamento de Trabajos Finales de Graduación, esto con el objetivo de: 1) adecuarlo a las necesidades presentes, 2) precisar en lo que a modalidades de TFG y requisitos se refiere 3) saldar algunas imprecisiones (por ejemplo lo señalado para las Memorias de los Seminarios en relación con que sus conclusiones no están necesariamente subordinadas a exigencias metodológicas). Por ello se celebra que en este momento exista una propuesta de nuevo Reglamento de Trabajos Finales de Graduación en consulta y se considera que la experiencia de las y los miembros de las Comisiones puede aportar mucho en la actualización de dicho reglamento.

\subsection{Apropiación colectiva de estrategias de mejora}

Como parte de los esfuerzos de integración y articulación emprendidos por la Facultad se ha identificado que una alternativa de solución consiste en colectivizar las guías, lineamientos e instructivos, de modo que la producción particular se vea enriquecida con la experiencia y sistematización de las otras unidades académicas, además se ha señalado que sería posible, incluso, uniformar dichos instructivos y guías.

Otra alternativa de solución vinculada con colectivizar los aprendizajes y estrategias implementadas por algunas unidades que han ideado fórmulas novedosas, es la de avanzar desde el diseño mismo del TFG en la identificación de los y las posibles integrantes del Comité Asesor, de modo que se aumente su vínculo y compromiso con las ideas y propuestas de las y los estudiantes. Lo mismo aplica para la relación directa que algunas unidades académicas han creado (Ciencias políticas y Comunicación por ejemplo) entre las y los docentes que imparten los cursos preparatorios a los TFG y la composición de la Comisión.

\subsection{Promoción de la interdisciplinariedad}

En la misma línea de argumentos, todas las unidades académicas de la Facultad reportan tener escasa o nula experiencia en el trabajo interdisciplinar, esta es sin duda una práctica que podría enriquecer al estudiantado y al personal docente, ampliando las visiones de mundo, promoviendo el intercambio de conocimientos, generando o potenciando las habilidades para el trabajo en equipo, el pensamiento y la construcción colectiva. El esfuerzo de integración y articulación puede materializarse en proyectos, seminarios, tesis o prácticas dirigidas que integren estudiantes de las diversas escuelas de la Facultad, esta es sin duda una solución plausible, que requiere la suma de voluntades para aventurarse a recorrer una ruta prácticamente inexplorada.

\subsection{Creación de líneas y temas de investigación en correspondencia con los desafíos contextuales atinentes a las ciencias sociales}

Una alternativa de solución identificada para los retos supracitados en los acápites 4.4 y 4.5 es la creación de líneas de investigación atinentes a los derroteros que enfrentan las ciencias sociales en la actualidad, la articulación de los TFG con los diversos institutos y centros de investigación vinculados con la Facultad, haciendo una lectura cercana del entorno nacional y sus desafíos contextuales es no sólo una solución posible, sino una necesidad impostergable.

\section{Referencias bibliográficas}

Universidad de Costa Rica, Vicerrectoría de Docencia: Circular VD-C-16-012.

Universidad de Costa Rica, Facultad de Ciencias Sociales: Taller de Comisiones de Trabajos Finales de Graduación 09 de abril de 2013. Universidad de Costa Rica, Facultad de Ciencias Sociales: Taller de Comisiones de Trabajos Finales de Graduación 24 de abril de 2013. Reglamento de Trabajos Finales de Graduación, Universidad de Costa Rica (Aprobado en sesión 2713-17, 04/08/1980. Publicado como anexo 1 del acta respectiva) 
